\title{
Research on city Trust Evaluation Model Based on Grid Computing and Context Awareness Xiangjun $\mathrm{Pu}$ \\ School of Urban Design, Wuhan University, Wuhan, China \\ Corresponding E-Mail:puxjt@126.com
}

Keywords: grid computing; trust evaluation model; context-aware;

\begin{abstract}
The current evaluation mechanism of city confidence based on grid computing is not perfect, which will greatly affect the efficiency and the grid resources' sharing. On the base of the traditional city model of grid computing, in this paper we propose a context-aware control and city trust evaluation model, estimating through the context similarity, effectively estimating unfamiliar entities trust and providing incentive punishment mechanism based on the actual transactions. Resource sharing is the main features of grid. Grid resource management based on the economy computing promotes the sharing of grid resources from many perspectives. These management methods are based on certain precondition, which assumes the resources' transaction parties in grid market are reliable.
\end{abstract}

\section{Introduction}

This paper also proposed grid computing city architecture and evaluation process based on trust, reducing the impact from the false service of unfamiliar entity on the trust evaluation model.

On this base people provide a variety of trust evaluation methods to achieve trust evaluation and management of entities. The main findings include trust evaluation based computing collaboration, context-aware inter-operable trust evaluation model, trust evaluation model grid services, trust-based evaluation of resource trading model. These trust evaluation model focuses on considering the validity of the trust, but lack of attention to the reliability of the grid entity trust evaluation when in the implementation. Due to the distribution of the grid system, heterogeneous and dynamic, the grid transactions often occur between unknown entities. Due to the strangeness of grid entities, the reliability of trust evaluation model in the actual run-time is reduced. To solve these problems, this paper presents a relatively reliable trust evaluation model in grid computing economy. When doing the trust evaluation on trustee, the trust-maker will enhance the accuracy of the evaluation trust between the strange entities by using trusted context and propose a mechanism for appropriate incentives and penalties trading entity thereby enhance the stability and reliability of trust evaluation model.

\section{Context-aware trust Evaluation Model}

\section{Context-aware}

The most essential feature of pervasive computing is transparent, mainly refers to whether the interaction between the user and the computer will be perceived. Just like the human interaction, people often intentionally or unintentionally use the common knowledge, atmosphere, context, environmental conditions and other contextual information to reduce the display interaction and improve the efficiency of interaction; a lot of context information in ubiquitous computing environment can also reduce or avoid the interaction between people and machine and among machines. This interaction is exactly an important way to achieve transparent interaction, and has thus become an independent field of study - context-aware computing. By perceiving the information available in the environment, context-aware meter automatically autonomously does reasoning, makes decision and calculates, thus greatly reducing the level required to participate, and achieving transparent interaction. Dey defines context as a set of all the available information of describing. This definition does not cover all aspects of the environment, such as: structural environment, areas, scope, nature, and its control function. 


\section{Trust Evaluation Model}

This article adopts Farag Azzedin's definition of trust: trust is the firm belief in the ability of an entity. Here embodied by trust, confidence is bound to a value of $[0,1]$, the greater the trust is, the more credible it will be. The change of trust depends on the physical behavior and adapts to the specific circumstances and time. Users and service providers need to make the evaluation after the transaction completes. Individual evaluation result made by the user affects the current trust of service providers. Similarly, the evaluation made by service provider also affects the user's current trust. The evaluation value generated in the direct transaction between Users and service providers is defined as direct trust. Usually at the end of the transaction, its size depends on the satisfaction of trading entity on transactions. If the user and the service provider have no direct transaction history, then the recommendation trust value on the transaction entity gotten through the recommender transmission will be defined as an indirect trust of service provider (user). The trust between service providers and users reviews first directly depends on their direct trust. If they have no trading history, we need to evaluate them by indirect reference. Trust evaluation model is shown in Figure 1.

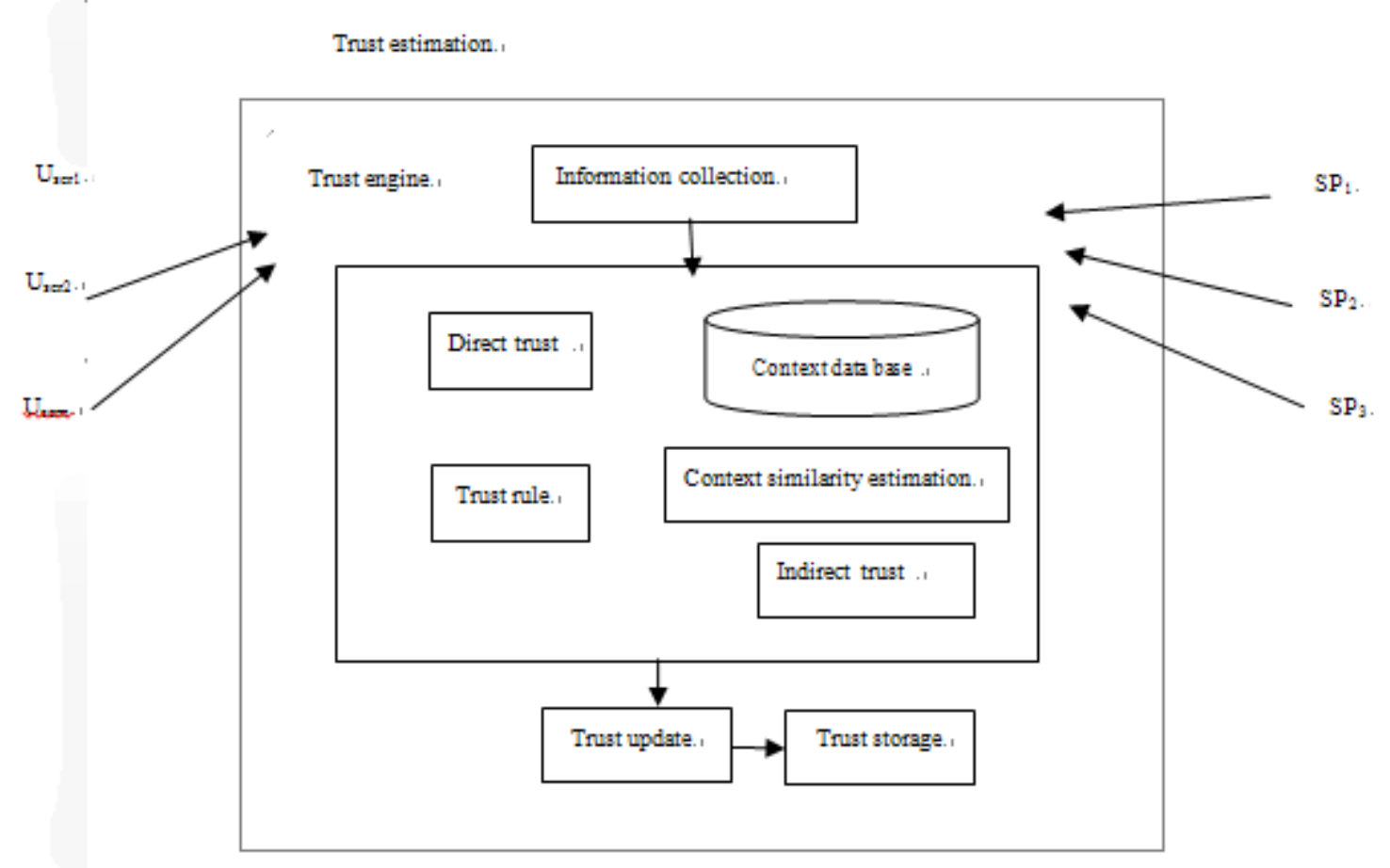

Figure 1 Trust evaluation model

Trust evaluation model contains information collection, Trust engine, trust update and trust store. Information collection module is responsible for collecting information about the trading transactions satisfaction, about trust of users (service provider) recommended to the service provider (user), about the context of recommender and valuator. Trust updating module is responsible for updating the trust of grid entity. Trust storage module is responsible for storing the trust of grid entity. Trust engine module is responsible for computing the trust of transaction entity.

Trust rule is to store trust evaluation rules. Direct trust computes the direct trust of trading entity on the basis of trust evaluation rules. The context database is used to store the context information of recommender and valuator. Context similarity estimation is responsible for estimating the similarity between the recommender context and the valuator context. Indirect trust calculates indirect credibility trading entity based on context similarity. As regard to the unreliability of trust estimation cause by the strangeness of grid entity, this paper introduces the similarity to quantify the context relation between recommender and evaluator and calculates the approximate value of corresponding context, and then improves the accuracy of the indirect trust and enhances the reliability of trust evaluation model in grid trading. 
The defined trust context refer to all the information that in the environment of a certain grid entity. Keywords are to describe entity context, that's to say $C_{i}$ is represented by $K_{\text {KeyWordSet }}$ (the $_{C_{i}}$ (the collection of keywords). The keywords in KeyWordSet $C_{C_{i}}$ have different weights.

On the condition of no trading history between $U_{\mathrm{j}}$ (user) and $S P_{i}$ (service provider), $C_{i}$ is the context for $U_{\mathrm{j}}$ and $S P_{i}$, the credibility about $S P_{i}$ that $R e c_{r}$ (recommender) recommend to $U_{\mathrm{j}}$ is $R\left(\operatorname{Rec}_{\mathrm{r}}, U_{j}, S P_{i}, C_{r}\right), C_{i}$ is the trading context between $\operatorname{Rec}_{r}$ and $S P_{i} \cdot C_{i}$ is presented as $K W S C_{i}=\left\{K_{1}, K_{2}, \cdots K_{i}\right\}$, the weight of each keyword in this collection is $\mathrm{k}_{i 1}, k_{i 2}, \cdots k_{i i} \cdot C_{r}$ is presented as $K W S C_{\mathrm{r}}=\left\{K_{1}, K_{2}, \cdots K_{m}\right\}$ and its weight is $\quad \mathrm{k}_{r 1}, k_{r 2}, \cdots k_{r_{m}}$.

This paper adopts Euclidean algorithm to compute the similarity $\left(S\left(C_{i}, C_{r}\right)\right)$ between $C_{i}$ and $C_{r}, \quad S\left(C_{i}, C_{r}\right)=1-\frac{1}{\sqrt{n}}\left[\sum_{h=0}^{n}\left(\alpha_{h}\left(k_{i h}-k_{r h}\right)^{2}\right)\right]^{\frac{1}{2}}, \mathrm{n}$ is the keywords number of the union of KeyWordSet $_{C_{i}}$ and KeyWordSet $C_{r}$. If $K_{h}$ in KeyWordSet $C_{C_{i}}$ can also find corresponding keyword in KeyWordSet ${ }_{C r}$, then $\alpha_{h}$ is 1 , otherwise it will be 0 .

\section{The estimation of context aware}

Let's assume that the closer between $C_{i}$ and $C_{r}$, the higher of credibility on users will be, and then the similarity between $C_{i}$ and $C_{r}$ can be regarded as the support of recommending credibility. When $S\left(C_{i}, C_{r}\right)<\mu$ ( $\mu$ is the recommending accuracy value), then the recommending credibility of the lower context similarity will be not $\mathrm{OK}$ and be filtered. If $S\left(C_{i}, C_{r}\right)>\mu$, then the recommending credibility is OK and no need to filter.

Assume that the recommending collection is $\operatorname{Re} c=\left\{\operatorname{Re} c_{1}, \operatorname{Re} c_{2}, \cdots \operatorname{Re} c_{n}\right\}$, and then the credibility recommended by $R \mathrm{Rc}_{r}$ will be shown as $R\left(\operatorname{Re} c_{r}, U_{j}, S P_{i}, C_{r}\right)$. The relatively credible credibility after filtering will be $R\left(\operatorname{Re} c_{r}, U_{j}, S P_{i}, C_{1}\right), R\left(\operatorname{Re} c_{r}, U_{j}, S P_{i}, C_{2}\right), \ldots R\left(\operatorname{Re} c_{r}, U_{j}, S P_{i}, C_{\mathrm{p}}\right)$.

When there is no direct trading history between users and service provider, the credibility of trading entity is $T=\frac{1}{p} \sum_{l=1}^{p}\left(S\left(C_{i}, C_{r}\right) \cdot R\left(E_{l}, U_{j}, S P_{i}, C_{l}\right)\right), \mathrm{p}$ is the number of relatively credible credibility after filtering and should not be zero. If the recommender's collection is null, then we can regard the trading entity as a new one in the grid environment, and set the trust of trading entity 0.5 ; if not, but the recommending credibility number after filtering is 0 , then it still be 0.5 .

\section{Incentive punishment mechanism of trading entity trust}

The incentive punishment mechanism can encourage users and service provider to trade more credibly. During the actual trading, if the service provider fulfills the task ahead of time, then we can inspire them by properly enhancing their trust. If the user saves the estimated cost, then we can inspire them by enhancing the users' credibility. If service provider is late to finish task, their credibility will be lowered down, and so do the user. Assume that the increment of incentive and punishment is $I$, and then the steps will be below:

As to trading time, if $t_{\text {pre.sp }}>t_{\text {real }}$, then $I=\eta_{1} *\left(t_{\text {presp }}-t_{\text {real }}\right) / t_{\text {presp }}$.

$$
\text { If } t_{\text {pre.sp }}<t_{\text {real }} \text {, then } I=\xi_{1} *\left(t_{\text {pre.sp }}-t_{\text {real }}\right) / t_{\text {pre.sp }} \text {. }
$$

As to trading cost, if $P_{\text {pre.u }}>P_{\text {real }}$, then $I=\eta_{2} *\left(P_{\text {pre.u }}-P_{\text {real }}\right) / P_{\text {pre.u }}$.

If $P_{\text {pre.u }}<P_{\text {real }}$, then $I=\xi_{2} *\left(P_{\text {pre.u }}-P_{\text {real }}\right) / t_{\text {pre.u }}$. 
$t_{\text {presp }}$ is the time that service provide promise to fulfill the task before trading, $t_{\text {real }}$ is the actual completing time. $P_{\text {pre.u }}$ is the estimated cost by users before trading, and $P_{\text {real }}$ is actual cost in trading. The punishment on grid trading entity is greater than its benefits, which also conforms to people's opinion on trust, $\eta_{1}, \eta_{2}, \xi_{1}, \xi_{2} \in[0,1], \eta_{1}>\xi_{1} \eta_{2}>\xi_{2}$ and $\eta_{1}-\xi_{1}, \eta_{2}-\xi_{2} \in(0,0.2]$

\section{Conclusion}

This paper proposes a healthy trust evaluation control model in grid computing economy. This model controls the reliability of evaluation on the base of context similarity. From the perspective of the whole city architecture and evaluation process, it has higher accuracy and intelligence and lays a solid foundation for the future improvement.

\section{References}

[1] Paul R, Yen I-Ling, Bastani F.An ontology-based integrated assessment framework for high-assurance systems[C]//The IEEE International Conference on Semantic Computing.2012.386-393.

[2] Grandison T.Trust management for internet applications[D].2011.

[3] Gruber T R.A translation approach to portable ontology specifications, Technical Report KSL 92-71[R].Knowledge System Laboratory, 2012.

[4] Ouyang Jian-quan, Ding Bo, Wang Huai-min.Component based context model[C]//The Ninth International Conference on Web-Age Information Management, 2008, 72: 569-573.

[5] Truong Binh-An, Lee Young-Koo, Lee Sung-Young.Modeling and reasoning about uncertainty in context-aware systems[C]// The IEEE International Conference on e-Business Engineering.2005.386-393.

[6] Gao J, Zhu E Y, Shim S.Monitoring software components and component-based software[C]//The 24th Annual International on Computer Software and Applications Conference.2000.403-412. 